\section{Vitamin D Deficiency in a Cohort of Patients with Systemic Scleroderma from the South of Spain}

\section{To the Editor:}

We read with interest the work by Vacca, et $a l^{1}$, who concluded that vitamin D deficiency is very common in patients with systemic sclerosis ( $\mathrm{SSc}$ ). We studied the prevalence of vitamin D deficiency and insufficiency and its possible relation to different clinical manifestations of the disease and to bone mineral density (BMD), in a cohort of patients with systemic sclerosis (SSc) from an autoimmune systemic disease unit in Southern Spain. We reviewed the clinical histories of 63 patients who attended our clinic between March and April 2009. Age, sex, height, weight, body mass index (BMI), years of development of disease, subtype of SSc, clinical manifestations, renal function tests, autoantibodies, type of treatment, vitamin D levels, $t$ score, and $\mathrm{z}$ score were gathered from all patients. A t score of -1 SD defined osteopenia and -2.5 SD defined osteoporosis.

We enrolled 48 patients in the study, for whom results of bone densitometry had been obtained. All patients were women, with a median age 59.1 \pm 11.7 years; $75.8 \%$ were postmenopausal.

Patient characteristics: the average for BMI was $27.7 \pm 5 \mathrm{~kg} / \mathrm{m}^{2} ; 91.5 \%$ had limited scleroderma (lcSSc) and $8.5 \%$ had diffuse scleroderma (dSSc); $89.4 \%$ had positive antinuclear antibodies, $49 \%$ positive anticentromere antibodies, and $6.4 \%$ positive anti-Scl-70 antibodies; $14.9 \%$ of patients were taking steroids, $17 \%$ immunosuppressive therapy, and one patient had received rituximab.

Vitamin D deficiency $(<30 \mathrm{ng} / \mathrm{ml})$ was found in $81 \%$ of the patients and insufficiency $(<10 \mathrm{ng} / \mathrm{ml})$ in $9.5 \%$, despite the fact that $60.41 \%$ of our patients were receiving "conventional" vitamin D supplements (cholecalciferol $800 \mathrm{IU} /$ day). In regard to BMD, $72.9 \%$ had low BMD $(47.9 \%$ osteopenia and 25\% osteoporosis); in the lumbar spine, $43.8 \%$ presented osteopenia and $16.7 \%$ osteoporosis; in the femoral neck $47.9 \%$ had osteopenia and $12.5 \%$ osteoporosis. Moreover $29.2 \%$ of patients presented a $\mathrm{Z}$ score $<-1$ SD (lumbar spine 16.7\%, femoral neck 22.9\%). Patients with vitamin D deficiency did not show a lower BMD. Indeed, there were no significant differences between patients with low and normal BMD in the variables studied, except BMI $\left(29.51 \pm 4.84\right.$ vs $26.48 \pm 4.27 \mathrm{~kg} / \mathrm{m}^{2}$, respectively; $p=0.04)$. The patients taking steroids did not show a major rate of osteoporosis/osteopenia. Neither were there any differences for BMD between patients with and those without calcinosis.

We found no significant correlation between vitamin D levels and different clinical variables, including systolic pulmonary artery pressure (sPAP), measured by Doppler echocardiography, and the presence or absence of lung fibrosis.

It is well known that the prevalence of deficient vitamin D levels is high, even in Mediterranean countries. In Granada, a sunny Andalucian city ( $37^{\circ} 11^{\prime}$ " north latitude) with 3016 hours of sun per year, we found levels of vitamin $\mathrm{D}<15 \mathrm{ng} / \mathrm{ml}$ in $39.1 \%$ of postmenopausal women, $70.7 \%$ of patients with inflammatory bowel disease, and $44.2 \%$ of corticosteroids-dependent asthmatic patients ${ }^{2}$. And from our study, as from Vacca, et $a l^{1}$, it seems that standard vitamin D supplements are insufficient to obtain adequate serum levels of the hormone.
Regarding BMD, some researchers have proposed that SSc could be a risk factor for osteoporosis ${ }^{3}$. In addition to the situation of chronic inflammation, other different factors related to low BMD (inactivity, malabsorption, renal insufficiency, drug use) influence these patients. Nevertheless, published studies are not conclusive and present contradictory information $^{4}$. The prevalence of low BMD (osteopenia-osteoporosis) in our patients with SSc was very high. In regard to SPAP, we found no correlation with vitamin D levels, probably due to the number of the patients, as vitamin D levels correlate with BMD in the general population ${ }^{5}$.

In summary, vitamin D deficiency in SSc seems to be high in different European populations, but studies with a greater number of patients are required to identify the exact role of vitamin D deficiency not only in development of osteopenia, but also in different manifestations of the disease, including sPAP, disease activity, and presence of lung fibrosis. Recently it was found that progression of fibrosis may be influenced by vitamin $\mathrm{D}$ deficiency decreasing the expression of antifibrotic factors ${ }^{6}$; moreover, if the study conclusions warrant levels of vitamin D > $30 \mathrm{ng} / \mathrm{ml}$, we will need to administer vitamin $\mathrm{D}$ in higher than the usual doses.

\section{RAQUEL RIOS FERNÁNDEZ, MD; CONCEPCIÓN FERNÁNDEZ} ROLDÁN, MD; JOSE LUIS CALLEJAS RUBIO, MD; NORBERTO ORTEGO CENTENO, MD, Autoimmune Diseases Unit, Hospital Clínicosan Cecilio, Avd Dr. Oloriz sn, Granada, Spain. Address correspondence to Dr. R Fernández; E-mail: rriosfer@hotmail.com

\section{REFERENCES}

1. Vacca A, Cormier C, Piras M, Mathieu A, Kahan A, Allanore Y. Vitamin D deficiency and insufficiency in 2 independent cohorts of patients with systemic sclerosis. J Rheumatol 2009;36:1924-9.

2. Mezquita P, Muñoz M, López F, Martínez Martín N, Conde Valero A, Ortego Centeno N, et al. Prevalence of vitamin D deficiency in populations at risk for osteoporosis: impact on bone integrity. Med Clin (Barc) 2002;119:85-9.

3. Souza RB, Borges CT, Takayama L, Aldrighi JM, Pereira RM. Systemic sclerosis and bone loss: the role of the disease and body composition. Scand J Rheumatol 2006;35:384-7.

4. Loucks J, Pope JE. Osteoporosis in scleroderma. Semin Arthritis Rheum 2005;34:678-82.

5. Boonen S, Rizzoli R, Meunier PJ, Stone M, Nuki G, Syversen U, et al. The need for clinical guidance in the use of calcium and vitamin $\mathrm{D}$ in the management of osteoporosis: a consensus report. Osteoporos Int 2004;15:511-9.

6. Artaza JN, Norris KC. Vitamin D reduces the expression of collagen and key profibrotic factors by inducing an antifibrotic phenotype in mesenchymal multipotent cells. J Endrocrinol 2009;200:207-21

J Rheumatol 2010;37:6; doi:10.3899/jrheum.091143 